\title{
CONTACT BOUNDARIES OF HYPERSURFACE SINGULARITIES AND OF COMPLEX POLYNOMIALS
}

\author{
CLÉMENT CAUBEL \\ Département de Mathématiques, Université de Cergy-Pontoise \\ 95033 Cergy-Pontoise, France \\ E-mail: caubel@math.u-cergy.fr \\ MIHAI TIBĂR \\ Mathématiques, UMR-CNRS 8524, Université de Lille 1 \\ 59655 Villeneuve d'Ascq, France \\ E-mail: tibar@agat.univ-lille1.fr
}

\begin{abstract}
We survey some recent results concerning the behavior of the contact structure defined on the boundary of a complex isolated hypersurface singularity or on the boundary at infinity of a complex polynomial.
\end{abstract}

1. Introduction. Let $f:\left(\mathbb{C}^{n+1}, \mathbf{0}\right) \rightarrow(\mathbb{C}, 0)$ be a germ of holomorphic function having an isolated singular point at the origin. Its boundary $\mathcal{B}_{\mathbf{0}}(f)$ is the intersection of the hypersurface $V(f):=f^{-1}(0)$ with a small sphere $S_{\varepsilon}$ centered at the origin, of radius $\varepsilon>0$. This is a closed oriented $(2 n-1)$-dimensional smooth manifold, which does not depend on $\varepsilon \ll 1$ up to isotopy. If we consider its embedding in the sphere $S_{\varepsilon}$, that is, the link $\mathcal{L}_{\mathbf{0}}(f):=\left(S_{\varepsilon}, S_{\varepsilon} \cap V(f)\right)$, then it determines completely the topological type of $V(f)$ (see [Mi]). On the other hand, the natural CR-structure on $S_{\varepsilon} \cap V(f)$, defined by the maximal complex hyperplane distribution in its tangent bundle, determines its analytical type (see $[\mathrm{Sch}]$ ). Thus we see that the boundary equipped with some additional structure can encode a lot of information on the singularity.

If we consider a polynomial function $f: \mathbb{C}^{n+1} \rightarrow \mathbb{C}$ then we have to deal with more global objects. Replacing small spheres by large ones, the boundary at infinity of the

2000 Mathematics Subject Classification: 32S55, 53D10, 53D15, 32S50, 57Q45, 32G07, 58H15.

Key words and phrases: contact structure on the link, isolated hypersurface singularities, topology of polynomial functions, deformations of isolated singularities, deformations of polynomials.

The paper is in final form and no version of it will be published elsewhere. 
general fiber of $f$ can be defined. Its embedding in the large sphere or its naturally defined CR-structure also provide potentially interesting invariants of $f$.

We present here some recent results on an intermediate structure: the contact structure defined by the maximal complex hyperplane distribution. Section 2 collects the definitions and results in contact geometry that we use in the following.

In the local case, Varchenko [V] showed that this structure is an analytic invariant of the singularity; we review this work in Section 3. In the global case, we present the authors' construction [CT] of the contact boundary at infinity attached to some polynomial. We therefore have well-defined contact boundaries attached to isolated singularities or to complex polynomials.

These contact boundaries are analytic (respectively algebraic) invariants, but a priori not topological invariants. This motivates the study of their variation in topologically trivial families of hypersurface singularities or of polynomials.

Section 4 is devoted to recent results showing that the formal homotopy class (i.e., the most primitive contact invariant, see Example 2.5) of these contact boundaries is constant in the following types of families, provided $n>2$ :

1. topologically trivial families of isolated hypersurface singularities, [C1].

2. families of polynomials $f_{s}$ such that their general fibers are homotopy equivalent to a wedge of $n$-spheres and such that their $n$-th Betti numbers are constant in the family, $[\mathrm{CT}]$.

We end this survey by some remarks and questions in Section 5 .

\section{A reminder on contact and almost contact structures}

2.1. Families of contact manifolds. For generalities on contact structures, see e.g. [Bl] or [El2] for a more up-to-date overview.

Definition 2.1. A contact form on a $(2 n-1)$-dimensional manifold $M$ is a global 1-form $\alpha$ on $M$ satisfying the non-integrability condition $\alpha \wedge(d \alpha)^{n-1}>0$. A contact structure on $M$ is the hyperplane distribution defined by a contact form. The notions of contactomorphism or of contact isotopy are naturally defined.

EXAmple 2.2. Any smooth level set of a strictly plurisubharmonic function on a complex manifold admits a natural contact structure, given by the maximal complex distribution in its tangent bundle (see e.g. [El1] for more details about contact structures and plurisubharmonic functions).

We now give the most general criterion for two contact structures to be isotopic.

Theorem 2.3 (J. W. Gray [G]). Let $\pi: M \rightarrow B$ be a smooth fiber bundle such that the fiber $M_{*}$ is a closed, oriented, odd-dimensional manifold. Suppose we are given a contact structure $\xi_{b}$ on each fiber $M_{b}$ which depends smoothly on the points b of the base. Then the contact manifolds $\left(M_{b}, \xi_{b}\right)_{b \in B}$ are all contact isotopic.

This theorem will be used in Section 3 to show that the contact manifolds we associate to isolated singularities or complex polynomials are well defined. 


\subsection{Homotopies of almost contact manifolds}

Definition 2.4. Let $M$ be a smooth closed oriented odd-dimensional manifold. An almost contact structure $\left({ }^{1}\right)$ on $M$ is a hyperplane distribution $\xi \subset T M$ endowed with a complex multiplication $J: \xi \rightarrow \xi$. An almost contact manifold is a manifold endowed with an almost contact structure. Finally, two almost contact manifolds $\left(M_{1}, \xi_{1}, J_{1}\right)$ and $\left(M_{2}, \xi_{2}, J_{2}\right)$ are almost contact homotopic if they are isotopic submanifolds of a manifold $W$ and if any isotopy between them carries $\left(\xi_{1}, J_{1}\right)$ on an almost contact structure on $M_{2}$ which is homotopic to $\left(\xi_{2}, J_{2}\right)$ in the space of almost contact structures on $M_{2}$.

ExAmple 2.5. Let $(M, \xi)$ be a contact manifold. Then any choice of a Riemannian metric on $M$ and of a contact form defines a complex structure on $\xi$ : the restriction of the 2 -form $d \alpha$ on $\xi$ is non-degenerate, so it gives a complex structure on $\xi$ in presence of the metric. While this almost contact structure is not well defined, its homotopy class in the space of all almost contact structures on $M$ is: it is called the formal homotopy class of the contact structure $\xi$. This class is easily seen to be invariant up to contact isotopy, and provides the most primitive global invariant of contact structures (see [El2]).

ExAmple 2.6. Any (real, cooriented) hypersurface $M$ in an almost complex manifold $(W, J)$ is naturally endowed with an almost contact structure: the complex hyperplane distribution $\left(\xi_{M}, J_{M}\right)$ is just the maximal complex subbundle $T M \cap J T M$ of the tangent bundle to the hypersurface equipped with the complex structure induced by $J$. Moreover, we have the following:

Proposition 2.7. Any two isotopic real hypersurfaces in an almost complex manifold are almost contact homotopic.

Sketch of proof. There are two equivalent ways to prove this. The first is to consider homotopy classes of almost contact structures on a $(2 n-1)$-dimensional oriented manifold as group reductions of its tangent bundle from $S O_{2 n-1}$ to $U_{n-1}$. Then in the context of this proposition, one builds a homotopy between the Gauss maps induced from the embeddings of the hypersurfaces in the almost complex manifold, which gives a homotopy of almost contact structures. This is the approach used in [C1].

The second way, used in $[\mathrm{CT}]$, is to identify almost contact structures with 2-forms of maximal rank when a Riemannian metric is fixed. One then chooses a Hermitian metric on the ambiant almost complex manifold and constructs a homotopy between the associated 2-forms on the two hypersurfaces.

This proposition means that if we define the formal homotopy class of a hypersurface in an almost complex manifold as the homotopy class of its almost contact structure, this formal homotopy class is an isotopy invariant. Of course, for a strictly pseudoconvex hypersurface (that is, a smooth level set of a strictly plurisubharmonic function) in a complex manifold, which is then also a contact manifold, the two notions of formal homotopy classes coincide.

This proposition will be used in Section 4 to show that the contact manifolds we associate to isolated singularities or complex polynomials stay in the same formal homotopy class in certain deformations.

$\left({ }^{1}\right)$ This definition differs slightly from the common one, see e.g. [Bl]. 


\section{Definition of the contact boundary in the local and global cases}

3.1. The local case: Varchenko's work. We summarize in this subsection the main results obtained by Varchenko in [V]. While it was known long before that the regular level sets of strictly plurisubharmonic functions on complex manifolds were natural examples of contact manifolds, it was the first time when this was used in the context of singularity theory.

Definition 3.1. Let $f:\left(\mathbb{C}^{n+1}, \mathbf{0}\right) \rightarrow(\mathbb{C}, 0)$ be a germ of holomorphic function with $\mathbf{0}$ as an isolated singular point. The contact boundary of $f$ is the boundary $\mathcal{B}_{\mathbf{0}}(f)$ of $f$ equipped with the contact structure defined by the maximal complex distribution of its tangent bundle.

The fact that this hyperplane distribution is indeed a contact structure is due to the strict plurisubharmonicity of the squared norm function $z \mapsto|z|^{2}$.

Proposition $3.2([\mathrm{~V}])$. The contact boundary of an isolated hypersurface singularity does not depend on the radius of the small sphere defining it up to contact isotopy. Moreover, it neither depends on the choice of analytic coordinates at $\mathbf{0}$ in $\mathbb{C}^{n+1}$.

Sketch of proof. Gray's Theorem 2.3 is used two times. First, the squared norm function restricted to $V(f)$ is a proper submersion over $\left(0, \varepsilon_{0}\right)$ for $\varepsilon_{0} \ll 1$ sufficiently small: this defines a family of contact manifolds, hence a contact isotopy. Second, since the group of analytic changes of coordinates is path connected, and because of Whitney's (b)-property satisfied by $(V(f) \backslash\{\mathbf{0}\},\{\mathbf{0}\})$ (see $[\mathrm{V}]$ for details), one can construct a convenient family of contact manifolds and then conclude.

This proposition shows that the contact boundary is a well-defined analytic invariant of the singularity defined by $f$.

Having shown this invariance, Varchenko applies it to quasi-homogeneous singularities. He proves that in this case the contact boundary is Sasakian (a certain integrability condition, see e.g. [Bl]), which implies that its odd Betti numbers are even up to half its dimension. This gives a strong restriction for a singularity to be topologically equivalent to a quasi-homogeneous one. Varchenko considers as examples Brieskorn's cusp singularities:

$$
f_{p, q, r}:(x, y, z) \mapsto x^{p}+y^{q}+z^{r}+x y z, \quad \frac{1}{p}+\frac{1}{q}+\frac{1}{r}<1 .
$$

Their boundaries satisfy $\operatorname{dim} H_{1}\left(\mathcal{B}_{\mathbf{0}}\left(f_{p, q, r}\right)\right)=1$, hence these singularities are not topologically equivalent to quasi-homogeneous ones.

3.2. The global case. Let us first take a complex algebraic set $V \subset \mathbb{C}^{n+1}$ having at most isolated singularities. It is proved in $[\mathrm{CT}]$ that, for large enough radius $R$, the intersection $S_{R} \cap V$ is a contact manifold, which is independent on $R$, up to contact isotopy. It turns out that instead of using spheres $S_{R}$, which are levels of the squared distance function, one can use the levels of some pseudo-convex rug function at infinity, i.e. a proper real polynomial map $\rho: \mathbb{C}^{n+1} \rightarrow \mathbb{R}_{\geq 0}$ which is strictly plurisubharmonic 
(see Remark 5.1 for the relation with the local case). Then the intersections $\rho^{-1}(R) \cap V$ do not depend on the choice of $\rho$ and of $R \geq R_{\rho} \gg 1$, up to contact isotopy. These observations yield a well-defined contact boundary of $V$ which we shall denote in the following by $\mathcal{B}_{\infty}(V)$.

Let now $f: \mathbb{C}^{n+1} \rightarrow \mathbb{C}$ be a complex polynomial function. It is well-known (see [Th], [Br]) that there exists a finite set $B_{f} \subset \mathbb{C}$ (which we shall suppose minimal) such that the restriction $f_{\mid}: f^{-1}\left(\mathbb{C} \backslash B_{f}\right) \rightarrow \mathbb{C} \backslash B_{f}$ is a locally trivial $\mathrm{C}^{\infty}$ fibration. Any value $t \notin B_{f}$ is called typical, as is the corresponding fiber $f^{-1}(t)$. The atypical values (i.e., those in $B_{f}$ ) are due not only to the critical points of the function $f$, but also to a certain bad asymptotic behavior at infinity.

It follows from the above discussion that any typical fiber $f^{-1}(t)$ has a well-defined contact boundary $\mathcal{B}_{\infty}\left(f^{-1}(t)\right)$. Moreover, we shall show how to define a generic contact boundary, independent on the fiber $f^{-1}(t)$, except for finitely many values of $t$.

Let $\rho: \mathbb{C}^{n+1} \rightarrow \mathbb{R}_{\geq 0}$ be a pseudo-convex rug function at infinity. We say that the fiber $f^{-1}\left(t_{0}\right)$ is $\rho$-regular-at-infinity if there exists a (small enough) disk $D_{\delta} \subset \mathbb{C}$ centered at $t_{0}$ and a (large enough) real $R_{\delta} \gg 0$ such that, for any $R \geq R_{\delta}$, the level $\rho^{-1}(R)$ is transversal to $f^{-1}(t)$ for all $t \in D_{\delta}$.

It follows from this definition that a $\rho$-regular-at-infinity fiber can have at most isolated singularities. By [T1], [T2, Prop. 2.6], if the fiber $f^{-1}\left(t_{0}\right)$ is non-singular and is $\rho$-regular-at-infinity for some $\rho$, then $t_{0}$ is a typical value of $f\left({ }^{2}\right)$.

Let $\operatorname{Reg}_{\infty} f$ denote the set of values $t \in \mathbb{C}$ such that the fiber $f^{-1}(t)$ is $\rho$-regular-atinfinity, where $\rho$ is some pseudo-convex rug function at infinity. Then the precise meaning of the notion of "generic contact boundary" of a polynomial $f$ is given by the following:

Theorem $3.3([\mathrm{CT}])$. Let $f: \mathbb{C}^{n+1} \rightarrow \mathbb{C}$ be a complex polynomial function. The contact boundary of a regular-at-infinity fiber $f^{-1}(t)$ does not depend, up to contact isotopy, on the choice of the value $t \in \operatorname{Reg}_{\infty} f$.

Sketch of proof. Let $d_{0}$ denote the squared distance function. We have proved in [T2, Cor. 2.12] that the set of values $t$ such that the fiber $f^{-1}(t)$ is not $d_{0}$-regular-at-infinity is a finite set. It then follows that the complement of $\operatorname{Reg}_{\infty} f$ in $\mathbb{C}$ is a finite set (which of course contains $B_{f}$ ).

We first reduce the problem to the case when $t_{1}, t_{2} \in \operatorname{Reg}_{\infty} f$ are both $d_{0}$-regular-atinfinity values. Then use a path $\Gamma$ from $t_{1}$ to $t_{2}$ such that $\Gamma$ consists of only $d_{0}$-regular-atinfinity values. For sufficiently large $R, f_{\mid \Gamma}: f^{-1}(\Gamma) \cap S_{R} \rightarrow \Gamma$ is a smooth fibration. One applies Gray's Theorem 2.3 to show that the contact boundaries of the fibers $f^{-1}\left(t_{1}\right)$ and $f^{-1}\left(t_{2}\right)$ are contact isotopic.

The above theorem defines an invariant which we call contact boundary of $f$ and denote by $\mathcal{B}_{\infty} f$. Let us remark that it does not depend on changes of coordinates in the automorphism group Aut $\mathbb{C}^{n+1}$ since the class of pseudo-convex rug functions at infinity is right-invariant by the action of Aut $\mathbb{C}^{n+1}$.

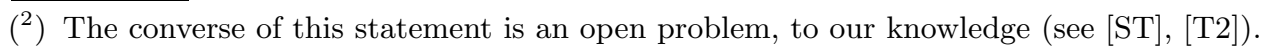




\section{Evolution of the contact boundary in numerically constant deformations}

4.1. The local case: invariance of the formal homotopy class in $\mu$-constant deformations. Let $f:\left(\mathbb{C}^{n+1}, \mathbf{0}\right) \rightarrow(\mathbb{C}, 0)$ be a holomorphic germ defining an isolated singularity at $\mathbf{0}$. In [C1], the first named author addresses the following question: how does the contact boundary vary in topologically trivial deformations $f$ ? A partial answer was obtained (see also Remark 5.2 on Eliashberg's question): the most primitive global invariant of the contact boundary is preserved in such deformations. More precisely, we have the following:

TheOrem $4.1([\mathrm{C} 1])$. Let $n>2$ and let $\left(f_{s}\right)_{s \in[0,1]}:\left(\mathbb{C}^{n+1}, \mathbf{0}\right) \rightarrow(\mathbb{C}, 0)$ be a smooth family of holomorphic function germs having an isolated singular point at $\mathbf{0}$. Suppose that all these germs are topologically equivalent. Then the contact boundaries $\mathcal{B}_{\mathbf{0}} f_{0}$ and $\mathcal{B}_{\mathbf{0}} f_{1}$ are almost contact homotopic.

Sketch of proof. We rely on the proof in [LR] of the Lê-Ramanujam theorem relating topological triviality and $\mu$-constancy of deformations. For small enough, well-chosen positive real parameters $s, t, \varepsilon_{0}, \varepsilon_{s}$, one can use Gray's Theorem 2.3 to show the following contact isotopies:

$$
\begin{aligned}
\mathcal{B}_{0} f_{0}= & f_{0}^{-1}(0) \cap S_{\varepsilon_{0}} \stackrel{\text { cont }}{\simeq} f_{0}^{-1}(t) \cap S_{\varepsilon_{0}} \stackrel{\text { cont }}{\simeq} f_{s}^{-1}(t) \cap S_{\varepsilon_{0}}:=M_{0} \\
& \mathcal{B}_{\mathbf{0}} f_{s}=f_{s}^{-1}(0) \cap S_{\varepsilon_{s}} \stackrel{\text { cont }}{\simeq} f_{s}^{-1}(t) \cap S_{\varepsilon_{s}}=: M_{s} .
\end{aligned}
$$

Denote by $W:=f_{s}^{-1}(t) \cap\left(B_{\varepsilon_{0}} \backslash B_{\varepsilon_{s}}^{\circ}\right)$ the piece of the fiber $f_{s}^{-1}(t)$ between the two spheres of radius $\varepsilon_{0}$ and $\varepsilon_{s}$. The same reasoning as in [LR] shows that, under our hypotheses, $W$ is a $h$-cobordism between $M_{0}$ and $M_{s}$. Since $n>2$, the two hypersurfaces $M_{0} \simeq \mathcal{B}_{0} f_{0}$ and $M_{s} \simeq \mathcal{B}_{0} f_{s}$ are isotopic in the complex manifold $W$. We then use Proposition 2.7 to conclude.

4.2. The global case: invariance of the formal homotopy class in $\gamma$-constant $V$-deformations. We shall call family of polynomials a family $f_{s}: \mathbb{C}^{n+1} \rightarrow \mathbb{C}$ depending smoothly on the parameter $s \in[0, \varepsilon]$, for some real $\varepsilon>0$. We have used in the local case the equivalence between the topological triviality and the $\mu$-constancy, which allowed us to conclude by using the $h$-cobordism theorem. In case of polynomial functions, even if we suppose that $f_{s}$ has isolated singularities, the total Milnor number is by far not enough to control the topology of $f_{s}$. A class of polynomials which turns out to be a good candidate for the analogue of the class of germs of isolated singularities is given in the following.

Definition 4.2. We say that a family of polynomials is a $V$-deformation of $f=f_{0}$ if, for all $s \in[0, \varepsilon]$,

(V) the typical fiber of $f_{s}$ is homotopy equivalent to a bouquet of spheres $\bigvee_{\gamma_{s}} S^{n}$.

If moreover the number $\gamma_{s}$ of spheres is independent on $s$, then we say that we have a $\gamma$-constant $V$-deformation.

The class of $\gamma$-constant $\mathrm{V}$-deformations has been considered in [T1], [T2] for proving a Lê-Ramanujam type result concerning the constancy of the monodromy at infinity. It 
is easy to see that if a $\mathrm{V}$-deformation is topologically trivial then it is $\gamma$-constant. The converse is not true, see [T2], [CT].

ExAmple 4.3. The polynomials with isolated $\mathcal{W}$-singularities at infinity in the sense of Siersma-Tibăr [ST] are examples of polynomials satisfying condition (V). This class includes the polynomials such that all their fibers are $\rho$-regular-at-infinity (for some $\rho$ ) and all polynomials of 2 complex variables with irreducible fibers.

Theorem 4.4 ([CT]). Let $n>2$ and let $\left(f_{s}\right)_{s \in[0, \varepsilon]}: \mathbb{C}^{n+1} \rightarrow \mathbb{C}$ be a $\gamma$-constant $V$-deformation of polynomial functions. Then the contact boundaries $\mathcal{B}_{\infty} f_{0}$ and $\mathcal{B}_{\infty} f_{1}$ are almost contact homotopic. In particular, this is the case in topologically trivial $V$-deformations.

Sketch of proof. It has been observed in [T1], [T2] that the arguments used in the local case by Lê-Ramanujam ([LR]) can be applied in case of polynomials satisfying the condition (V) when replacing the small spheres by large ones. In our context, we paraphrase the proof of Theorem 4.1 like this: for a smooth $d_{0}$-regular at infinity fiber $f_{0}^{-1}(t)$ and for a good choice of parameters satisfying $1 \gg R_{0}^{-1} \gg s \gg \delta \gg R_{s}^{-1}>0$, we get the following contact isotopies:

$$
\begin{gathered}
\mathcal{B}_{\infty} f_{0} \stackrel{\text { cont }}{\simeq} f_{0}^{-1}(t) \cap S_{R_{0}} \stackrel{\text { cont }}{\simeq} f_{s}^{-1}(t) \cap S_{R_{0}} \stackrel{\text { cont }}{\simeq} f_{s}^{-1}(t+\delta) \cap S_{R_{0}}, \\
\mathcal{B}_{\infty} f_{s} \stackrel{\text { cont }}{\simeq} f_{s}^{-1}(t+\delta) \cap S_{R_{s}} .
\end{gathered}
$$

The cobordism $W:=f_{s}^{-1}(t+\delta) \cap\left(B_{R_{s}} \backslash B_{R_{0}}^{\circ}\right)$ between the two contact boundaries is a product $h$-cobordism under our hypotheses, which allows us to conclude using Proposition 2.7. Notice that we were bound to pass from $t$ to $t+\delta$ in the base, since the $d_{0}$-regularity at infinity of $f_{s}^{-1}(t)$ is not guaranteed.

\section{Remarks and questions}

REMARK 5.1. For simplicity, we have defined in the local case the boundary of an analytic function germ by means of the squared norm function. But this choice may seem too restrictive: for instance, in [Lo] the boundary in defined by any rug function, that is, any proper real analytic function $\rho:\left(\mathbb{C}^{n+1}, \mathbf{0}\right) \rightarrow \mathbb{R}_{\geq 0}$ such that $\rho^{-1}(0)=\mathbf{0}$. This does not change the isotopy type of the boundary. Since we want to get a well-defined contact structure on the boundary, the good generalization in our context is the notion of pseudo-convex rug function, that is, a strictly plurisubharmonic rug function $\left({ }^{3}\right)$. This does not change the contact isotopy type of the contact boundary, which gives another proof of Proposition 3.2: the class of pseudo-convex rug functions is right invariant by the action of the group $\operatorname{Aut}\left(\mathbb{C}^{n+1}, \mathbf{0}\right)$ of analytic changes of coordinates. This is the approach we have used in the global case.

REMARK 5.2. In [El1], Eliashberg raises the following " $J$-convex $h$-cobordism problem": suppose we have a strictly plurisubharmonic function $f$ on a product $W=M \times[0,1]$, such that the two boundary components $M \times\{i\}, i=0,1$, are level sets of $f$. Is it possible to find another strictly plurisubharmonic function $g$ which coincides with $f$ on the boundary and without critical points?

$\left({ }^{3}\right)$ This was used by Ehlers, Neumann and Scherk in [ENS] for surface singularities. 
Were this true, the proof of Theorem 4.1 would improve to yielding that the contact boundary is invariant in topologically trivial deformations. In the global case too (Theorem 4.4) this would yield the invariance of the contact boundaries in $\gamma$-constant (and, in particular, topologically trivial) V-deformations.

REMARK 5.3. The results of Section 4 were proved by using the $h$-cobordism theorem, which excludes the surface case $n=2$. For normal surface germs, thanks to the plumbed structure of the boundary given by its topological type (see $[\mathrm{N}]$ ), one can show that the formal homotopy class of the contact boundary is in fact a topological invariant. This will appear elsewhere [C2].

REMARK 5.4. Following the preceding remark, one may ask in the general case if the formal homotopy class of the contact boundary is a topological invariant, which is $a$ priori a stronger condition than being an invariant of topologically trivial deformations. The answer is only known in the particular case when this boundary is diffeomorphic to the standard sphere $S^{2 n-1}$. This is yes, in the local case, by Morita's work [Mo]. Morita gives formulae expressing the formal homotopy class (which is then an element of $\left.\pi_{2 n-2}\left(S O_{2 n-1} / U_{n-1}\right)\right)$ in terms of the Milnor number $\mu$ and of the signature of the Milnor fiber, which are both topological invariants.

\section{References}

[Bl] D. E. Blair, Contact Manifolds in Riemannian Geometry, Lecture Notes in Math. 509, Springer, Berlin, 1976.

[Br] S. A. Broughton, Milnor number and the topology of polynomial hypersurfaces, Invent. Math. 92 (1988), 217-241.

[C1] C. Caubel, Structures presque de contact et singularités isolées, C. R. Acad. Sci. Paris Sér. I Math. 333 (2001), 339-342.

[C2] C. Caubel, Bord de contact des singularités de surfaces normales, in preparation.

[CT] C. Caubel, M. Tibăr, The contact boundary of a complex polynomial, Manuscripta Math. 111 (2003), 211-219.

[ENS] F. Ehlers, W. D. Neumann, J. Scherk, Links of surface singularities and CR space forms, Comment. Math. Helv. 62 (1987), 240-264.

[El1] Y. Eliashberg, Symplectic geometry of plurisubharmonic functions (with notes by Miguel Abreu), in: Gauge Theory and Symplectic Geometry, NATO Adv. Sci. Inst. Ser. C Math. Phys. Sci. 488, Kluwer, Dordrecht, 1997, 49-67.

[El2] Y. Eliashberg, Invariants in contact topology, in: Proceedings of the International Congress of Mathematicians, vol. II (Berlin, 1988), Doc. Math. 1998, Extra Vol. II (electronic), 327-338.

[G] J. W. Gray, Some global properties of contact structures, Ann. of Math. (2) 69 (1959), 421-450.

[LR] Lê Dũng Tráng, C. P. Ramanujam, The invariance of Milnor number implies the invariance of the topological type, Amer. J. Math. 98 (1976), 67-78.

[Lo] E. J. N. Looijenga, Isolated Singular Points on Complete Intersections, London Math. Soc. Lecture Note Ser. 77, Cambridge University Press, Cambridge, 1984. 
[Mi] J. Milnor, Singular Points of Complex Hypersurfaces, Ann. of Math. Studies 61, Princeton Univ. Press, Princeton, 1968.

[Mo] S. Morita, A topological classification of complex structures on $S^{1} \times \Sigma^{2 n-1}$, Topology 14 (1975), 13-22.

[N] W. D. Neumann, A calculus for plumbing applied to the topology of complex surface singularities and degenerating complex curves, Trans. Amer. Math. Soc. 268 (1981), 299-344.

[Sch] J. Scherk, CR structures on the link of an isolated singularity, in: Proceedings of the Vancouver Conference in Algebraic Geometry, CMS Conf. Proc. 6, Amer. Math. Soc., Providence, 1986, 397-403.

[ST] D. Siersma, M. Tibăr, Singularities at infinity and their vanishing cycles, Duke Math. J. 80 (1995), 771-783.

[Th] R. Thom, Ensembles et morphismes stratifiés, Bull. Amer. Math. Soc. 75 (1969), 240-284.

[T1] M. Tibăr, On the monodromy fibration of polynomial functions with singularities at infinity, C. R. Acad. Sci. Paris Sér. I Math. 324 (1997), 1031-1035.

[T2] M. Tibăr, Regularity at infinity of real and complex polynomial functions, in: Singularity theory (Liverpool, 1996), London Math. Soc. Lecture Note Ser. 263, Cambridge Univ. Press, Cambridge, 1999, 249-264.

[V] A. N. Varchenko, Contact structures and isolated singularities, Vestnik Moskov. Univ. Ser. I Mat. Mekh., 1980, no. 2, 18-21; English transl.: Mosc. Univ. Math. Bull. 35 (1980), no. $2,18-22$. 